\title{
The Effects of Tags on the Evolution of Honest Signaling
}

\author{
A. Pinar Ozisik \\ DEMO Lab, School of Computer Science, \\ Brandeis University, Waltham, MA \\ pinar89@brandeis.edu
}

\author{
Kyle I. Harrington \\ DEMO Lab, School of Computer Science, \\ Brandeis University, Waltham, MA \\ kyleh@cs.brandeis.edu
}

\begin{abstract}
In the study described here we examine the importance of social tags in the emergence and maintenance of signaling, using the Sir Philip Sydney Game. We use tags in the calculation of inclusive fitness for members in a finite population, and analyze their evolution under different population distributions. We support the claim that inclusive fitness theory may not be sufficient to explain the evolution of cooperation. While cooperativity through honest signaling is sometimes achieved with tag-based relatedness, we suggest that the importance of tag-based mechanisms may not simply be due to their role in kin selection.
\end{abstract}

\section{Categories and Subject Descriptors}

I.2.6 [Computing Methodologies]: Artificial IntelligenceLearning

\section{General Terms}

Theory

\section{Keywords}

Tags, Signaling, Coevolution, Population dynamics, Communication, Kin selection, Cooperation

\section{INTRODUCTION}

The emergence and maintenance of signaling has been a subject of interest in many fields including the social and biological sciences. Computational studies incorporate theory from these fields into studies of the evolution of communication; for an extensive review on this subject see [22]. Many forms of sociality rely on communication. Consider the specific form of sociality seen in eusocial organisms; eusocial organisms such as ants and bees establish reproductive castes and exhibit trophallaxis (food sharing) amongst other traits [23]. These organisms are known to communicate via

Permission to make digital or hard copies of all or part of this work for personal or classroom use is granted without fee provided that copies are not made or distributed for profit or commercial advantage and that copies bear this notice and the full citation on the first page. To copy otherwise, to republish, to post on servers or to redistribute to lists, requires prior specific permission and/or a fee.

GECCO'12 Companion, July 7-11, 2012, Philadelphia, PA, USA.

Copyright 2012 ACM 978-1-4503-1178-6/12/07 ...\$10.00. various signaling systems. The relationship between signaling and trophallactic behavior, altruistic food sharing, is of particular interest within the scope of this study.

Models of honest signaling have been developed and applied to food sharing between offspring and parents for mammals and birds [19, 12, 1], as well as insects [16]. In these models, variation in intensities of need trigger different begging behaviors on the part of offspring, while varying costs and benefits of food sharing trigger different donation behaviors on the part of the parents. Although some models of signaling are limited to the effect of intensities of need, signal, and donation costs [10], the majority of the studies that have been embraced within the biological literature also account for the relatedness between signaler and donor [19]. This relatedness is based upon an idea called, inclusive fitness [7], which has been one of the primary tools for explaining how such altruistic behavior could emerge. One of the key reasons that inclusive fitness theory has been widely used is the simplicity of Hamilton's rule

$$
r B>C
$$

where $r$ is the genetic relatedness of the recipient to the donor, $B$ is the benefit to the recipient, and $C$ is the cost of donation to the donor. However, the theory of inclusive fitness has recently been weakened by a number of counterarguments [15]. These counter-arguments include: a lack of mathematical relationship to population genetics, misinterpretation of other dynamics (such as spatial interactions) as the $r$ component of Hamilton's rule, and a failure to explain more than a subset of eusocial interactions.

Another topic of interest extending from this question includes social tags [9], which can be described as markings, arbitrary characteristics or phenotypic qualities enabling members in a population to recognize similarities among each other. In the past, there have been studies relating tags to the emergence of cooperation [17]. The underlying explanation for this relation comes from indirect reciprocity, described as the increased probability of receiving help from others when an individual helps a random member in their population. Additionally, in iterated games such as prisoner's dilemma and snowdrift, tags are known to promote cooperation $[5,4]$.

In the context of cooperation games, where there is a donor and recipient, cooperation involves donation and is measured as a function of donation rate $[17,20]$. In a broader sense, however, cooperation is the process by which individuals appearing to be selfish work together for an overall net gain in the system. While Nowak et al. suggest that the genetic relatedness component of inclusive fitness theory may 
(a) Payoff matrix.

\begin{tabular}{|l|r|r|}
\hline & Donate & Keep \\
\hline Potential donor & $1-d$ & 1 \\
\hline Signaler & 1 & $1-a$ \\
Thirsty & 1 & $1-b$ \\
Healthy & Signal cost $=c$ \\
\hline$m, \quad$ probability sig- \\
naler is thirsty & \multicolumn{2}{|l}{} \\
\hline
\end{tabular}

(c) Donor strategies.

(b) Sender strategies.

\begin{tabular}{|c|c|c|c|}
\hline ID & Signaler strategies & ID & Donor strategies \\
\hline $\mathrm{SH}$ & signal only if healthy & $D Q$ & donate only if no signal \\
\hline$S T$ & signal only if thirsty & $D S$ & donate only if signal \\
\hline$S N$ & never signal & $D N$ & never donate \\
\hline$S A$ & always signal & $D A$ & always donate \\
\hline
\end{tabular}

be mistaken for dynamics other than kinship (i.e. within spatial models), work on the emergence of collective behavior with tags in a spatial domain continues to lend credence to the importance of tag-based cooperation [21]. In this study, we investigate the effect of tags on the dynamics of signaling when coevolving trophallactic strategies and tagbased relatedness. In the context of trophallactic strategies in eusocial organisms, communal fidelity is crucial. In these type of societies deceptive signals are not beneficial to the system as a whole. Therefore, we consider whether a tagbased mechanism eases the reachability of honest signaling equilibria from a variety of initial conditions. We find that tags can only be successful for a subset of the conditions tested. For a majority of cases, instead of pushing the system towards honest signaling, tags favor uncommunicative behavior.

\section{SIR PHILIP SIDNEY GAME}

The Sir Philip Sidney (SPS) game was developed by John Maynard Smith as a model of costly signals [19]. It is an extensive form game between two players. The importance of costly signals is based upon Zahavi's handicap principle [24] which states that reliable signals are costly with respect to the signaler's ecological context. This cost is explicitly introduced as a fitness penalty in the SPS game.

The SPS game is played for a single round between two players: a signaler and a donor. The signaler may be in one of two states: thirsty or healthy. The probability of the signaler being thirsty is $m$. A thirsty signaler has a fitness of $(1-a)$, and a healthy signaler has a fitness of $(1-b)$. In all cases $a>b$. The strategy of the signaler specifies whether it signals in either, both, or neither states. It costs the signaler $c$ to transmit a signal. In response to receiving a signal, the donor decides whether or not to donate to the signaler. Donation comes at a cost, $d$, to the donor, but heals the signaler to a fitness of 1 . Furthermore, a relatedness term, $r$, is introduced which accounts for the opponent in the inclusive fitness of each player. Labels for signaler and donor strategies are listed in Tables $1(\mathrm{~b})$ and 1(c), respectively. For example, in a game between $S T$ and $D S$, if thirsty the signaler will transmit a signal and in response the donor donates. The signaler's fitness is $(1-c+r(1-d))$ and the donor's fitness is $(1-d+r(1-c))$. If the game was played between $S T$ and $D Q$, then if thirsty the signaler transmits a signal and the donor does not donate. The signaler's fitness is $(1-a-c+r)$ and the donor's fitness is $(1+r(1-a-c))$. The payoff matrix is shown in table 1 .

The key distinction between other games and and the SPS game is the use of inclusive fitness, adding the opponent's payoff weighted by a "relatedness" term, $r$. Relatedness ac- counts for the fact that if a player's opponent is related to the player, then benefits to the opponent also benefit the player. This makes it possible for cost-free signals to be evolutionarily stable when the game is played between relatives $[13,2]$. However, the ratio of cost of donation to the benefit of assistance limits the stability of these equilibria, hence it is concluded that cost-free signals may be stable when donation is inexpensive relative to the benefit of assistance [13]. In games modeling cooperation [17] the ratio of benefit to donation must be greater than 1 .

\subsection{Non-communicative Equilibria}

In this study we are interested in honest signaling. While there are multiple combinations of signaler and donor strategies that do not transfer information, we will be particularly interested in the $S N$ and $D N$ combination of strategies, because this pairing is a Nash equilirium that is always present in the SPS game. Bergstrom and Lachmann [1] have shown the $S N$ and $D N$ pair to be a Nash equilibrium if

$$
d>r(m a+(1-m) b) .
$$

Huttegger and Zollman [11] note that reversing the inequality leads to the $S N$ and $D A$ pair of strategies being a Nash equilibrium. They refer to these as "pooling equilibria."

\subsection{Signaling Equilibria}

One of most commonly studied type of equilibria in signaling games with costly signals is the signaling ESS, sometimes referred to as separating equilibria. In these equilibria the $S T$ and $D S$ strategies are dominant. Bergstrom and Lachmann [1] show this is a Nash equilibrium when

$$
a \geq c+r d \geq b \quad \text { and } \quad a \geq d / k \geq b .
$$

We will refer to this type of signaling equilibrium as the honest signaling equilibrium. We have previously investigated the emergence of this type of equilibria from noncommunicative origins [8].

\section{TAGS}

Tags are phenotypic characteristics enabling individuals to identify similarities between each other. In previous studies, the mechanics behind tags have included several different implementations. Some experiments used multidimensional tags, represented as bit strings with Hamming distance or exact tag matching $[3,6]$. Other alternatives use real-valued tags and tolerance levels to tags [17]. In Riolo's implementation each agent in the population has a tag value and a tolerance threshold. At each generation, every agent acts as a potential donor with a set number of recipients chosen at random. If the tag difference between a donor/recipient pair 


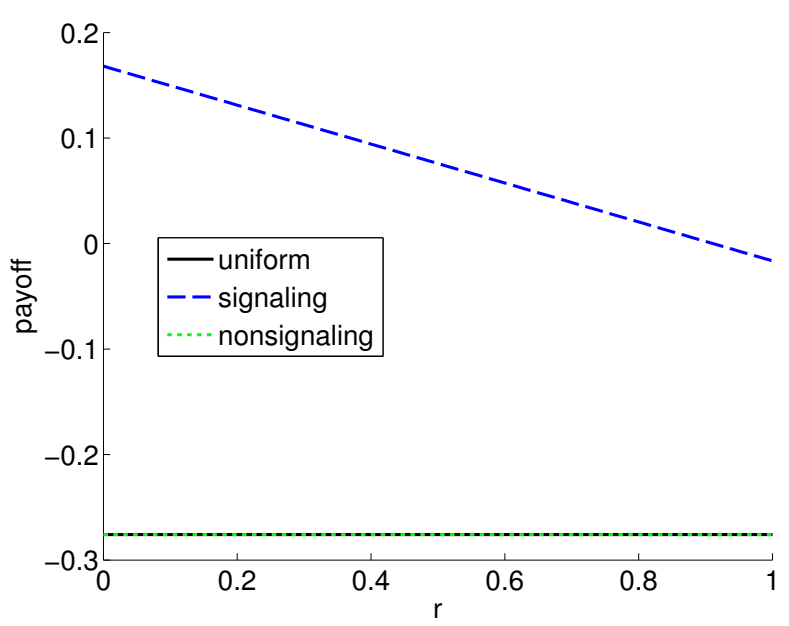

(a) Signaler Population

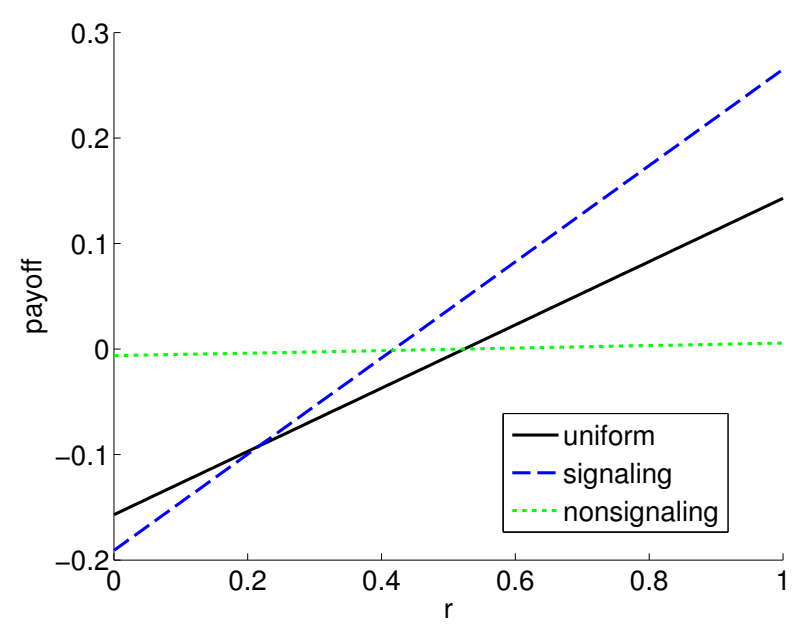

(b) Donor Population

Figure 1: Figure 1(a) shows the advantage/disadvantage of playing strategy $S T$ over $S N$ relative to different distributions of the donor population. Points above $x=0$ denote that $S T$ performs better, while points below $x=0$ denote that $S N$ performs better. Figure 1(b) shows the advantage/disadvantage of playing strategy $D S$ over $D N$ relative to different distributions of the signaler population. Points above $x=0$ denote that $D S$ performs better, while points below $x=0$ denote that $D N$ performs better. Note that maxiumum payoff can be achieved through $S T$ and $D S$.

is within the donor's tolerance threshold, they donate, paying cost $c$ and the recipient gains benefit $b$. Typical values for $b$ and $c$ are 1.0 and 0.1 , respectively. Selection is based upon randomly choosing two agents and giving the one with a higher score some number of offsprings. In Riolo's model, both tag values and thresholds coevolve with a mutation rate of 0.1. The game is symmetric such that each agent plays the role of the donor and the recipient. Spector and Klein extend Riolo's work to multi-dimensional real-valued tags $[20]$.

In our experiments, we use one dimensional real-valued tags. We are mainly interested in the coevolution of tags and player strategies. In the context of the SPS game, tags become relevant when we consider the relatedness term, $r$. It is used for inclusive fitness, where a player's score is the sum of their own payoff and the opponent's payoff weighted by $r$. This implies that if a player's opponent is highly related to the player through a high $r$ value, benefits to the opponent are more beneficial to the player. Nevertheless, the relatedness term has different implications from the donor's and signaler's perspectives. Donors prefer to help those they are closely related to, while signalers prefer to receive donations from those they are less related to. Other than this main distinction between signaling games and the SPS game, we also note that the players are either donors or signalers, represented as two separate populations in our study. In Riolo's tag based mechanism, players play both roles.

\section{EXPERIMENTS}

When evaluating finite populations we employ a simple genetic algorithm [14]. In both populations individuals are represented as integers between 1 and 4 representing the strategies listed in Tables 1(c) and 1(b). Strategies are mutated with a probability of 0.05 , and no crossover is used. Mutation is perfomed by replacing an individual with a randomly generated strategy. Each individual plays 50 games against randomly selected individuals from the opposing population, and the average score of these games is treated as the individual's fitness.

The populations are of size 100, and are evolved for 5,000 generations. The genetic algorithm is run on 25 parameter sets, with 100 repeats per set. Parameters are generated to satisfy the previously described conditions for honest signaling equilibria. Additionally, each parameter set is tested with a continuous model initialized with uniform population distributions, using the discrete time replicator equation [18]. The motivation behind this is to confirm the accessibility of reaching the honest signaling equilibria from a given parameter set. If a set of parameters does not lead to the honest signaling equilibria within 5,000 generations, another set of honest signaling parameters is tested. This process was iterated to produce the 25 parameter sets used in these experiments.

We run our parameters on the finite populations with 3 different population distributions. For a uniform distribution, we represent all strategies equally in the population. For a signalling distribution, we initialize the populations with primarily $S T$ and $D S$ (97\% of the population), and small fractions of the remaining strategies (1\%). For a nonsignaling distribution, the populations are initialized with non-signaler and non-donors (97\% of the population), and equally distributed strategies among the rest (1\%).

Tags are used when calculating the scores of individuals in the genetic algorithm. Each member of the two populations, signalers and donors, has a tag-value, $t \epsilon[0,1]$. When calculating the average score for a given donor/signaler pair at any time, $r$ is defined as $\left(1-\left|t_{D}-t_{S}\right|\right)$. Tags are mutated with a probability of 0.10 , and no crossover is used. Mutation is achieved by replacing an individual's tag with a randomly generated number from a uniform distribution.

Natural selection is applied via tournament selection. We 

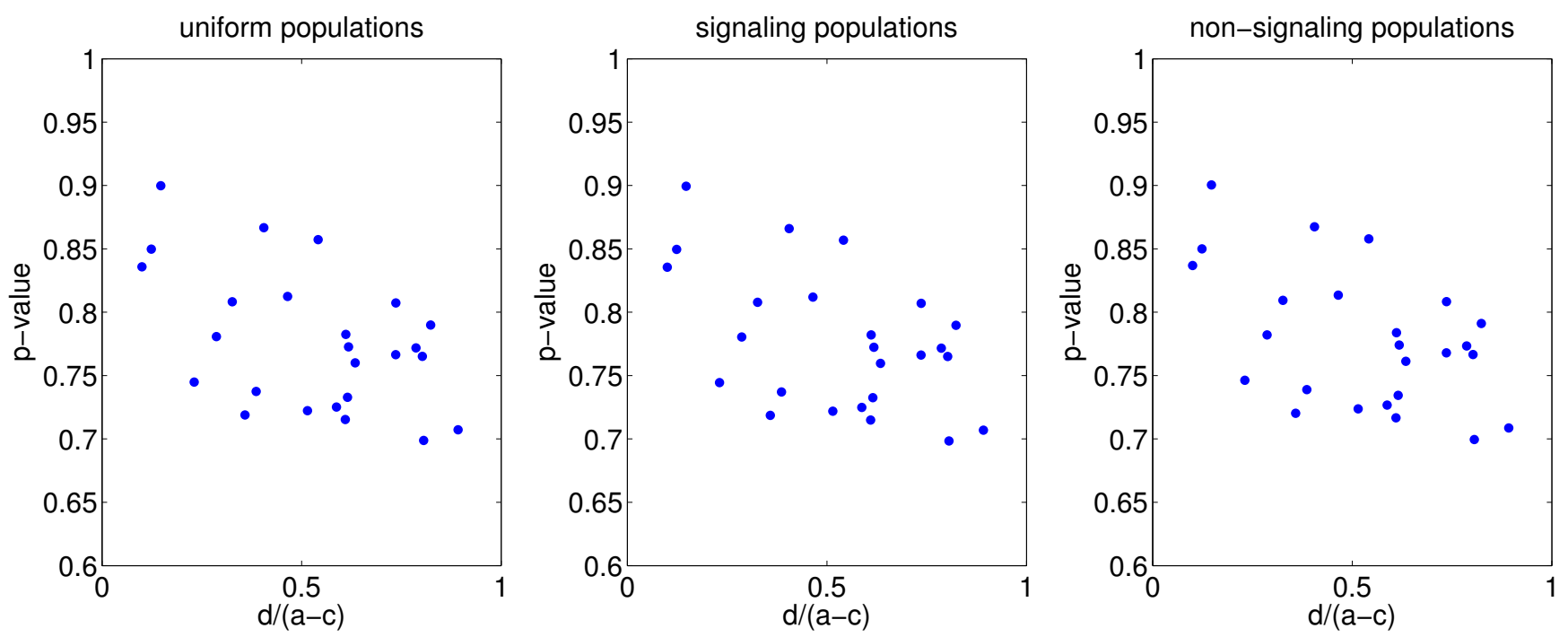

Figure 2: p-value of tag-based expected relatedness decreases as cost to benefit ratio increases.

ensure that all individuals have an equal opportunity to compete by constructing tournaments with random permutations of the population. For all experiments, we use a tournament size of 7 .

\subsection{Analysis of Relatedness in SPS}

Tags are known to contribute to the evolution of cooperation. To investigate whether tags lead to cooperation in the SPS game, we look at the payoff associated with specific strategies as a function of $r$. The payoff values shown in Figure 1(a) are calculated as

$$
\sum_{D} P_{D} \pi(S T, D)-\sum_{D} P_{D} \pi(S N, D)
$$

where $P_{D}$ is the fraction of donor strategy $D$ in the population, and $\pi(S, D)$ is the payoff associated with a signaler/donor pair. The first sum in this equation describes how $S T$ performs in relation to the four donor strategies. The second sum describes the same idea using the $S N$ strategy. The lines in Figure 1(a) represent the signaling, nonsignaling and uniform donor population distributions that each pure signaling strategy plays against. These population distributions are used for $P_{D}$, representing the distribution of a given donor strategy in the population. Therefore, Figure 1(a) represents the favorability of playing $S T$ relative to $S N$ against the three different population distributions. Figure 1(b) describes a similar property, where the favorability of playing $D S$ relative to $D N$ is calculated using different population distributions. Figure 1(b) shows

$$
\sum_{S} P_{S} \pi(S, D S)-\sum_{S} P_{D} \pi(S, D N)
$$

where $S$ represents a signaler strategy. The set of parameters used for these figures are $a=0.7552, b=0.3540, c=$ $0.4504, d=0.3140, m=0.6123$. These parameters were carefully chosen such that if $r$ falls within a given range, the honest signaling equilibrium can be reached.

Figure 1(a) suggests that it is more advantageous to play $S T$ than $S N$, when $D S$ is in the majority, because it has greater payoff. However, as $r$ increases, the payoff for the signaler decreases. For the other two population distributions, $S N$ has greater payoff. In fact, for both distributions we see identical lines. This can be explained by the equal distribution of donating strategies for both. When the distribution of the population is composed mainly of strategy $D N$, Figure 1(b) indicates that $D N$ has slightly higher payoff than $D S$. Nevertheless, for the other two population distributions the lines cross $x=0$, suggesting that $D S$ overtakes $D N$ when $r$ crosses specific threshold values. This suggests that with the evolution of a higher value for $r$, payoff can be maximized for the donors when the signalers are uniformly distributed or when $S T$ is the major strategy. Therefore, we hypothesize that tag values will evolve such that the score for the donor and signaler are maximized. This can occur at the intersections points of the two figures. We assume that signalers and donors will evolve tag values such that those intersection points are targeted. However, in a broader sense, we can say that the signaling equilibria are likely to emerge as $S T$ and $D S$ have maximal payoff against the opponent strategies in the long run.

For a cooperative system to emerge, the benefit to both donors and signalers needs to lead to mutual gain. However, as $r$ decreases, the payoff for $S T$ decreases. On the other hand, as $r$ increases, the payoff for $D S$ decreases. Thus there is a conflict of interest between the two strategies. Game theoretic analyses have derived a set of conditions under which these two strategies can coexist. Yet, if the value of $r$ is evolved via tags, then this conflict of interest may lead to behavior akin to pursuer-evader dynamics.

\subsection{Evolution of Strategies in SPS}

We generate 25 parameter sets, including an optimal $r$, for honest signaling equilibria to emerge. In this experiment, we do not change $r$ and keep it constant for all repeats of each set. We disable tag mutation and only let strategies evolve. By assigning a tag value of 1 to all donors and a tag value of $1-r$ to all signalers, we make sure that $\left(1-\left|t_{D}-t_{S}\right|\right)$ is the optimal $r$ for signaling. Figures 3(a) and 3(b) show the distribution of strategies for signalers and donors, respectively, 


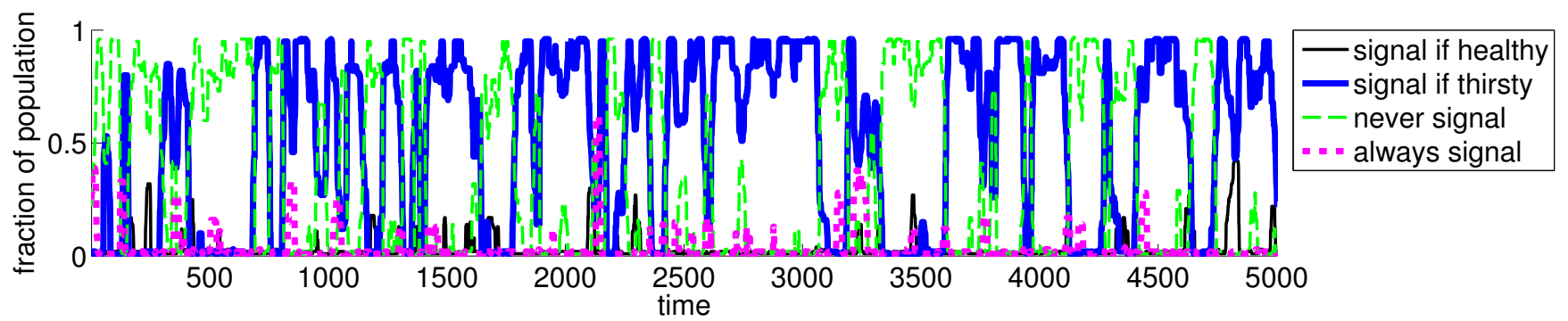

(a) Signaler Population

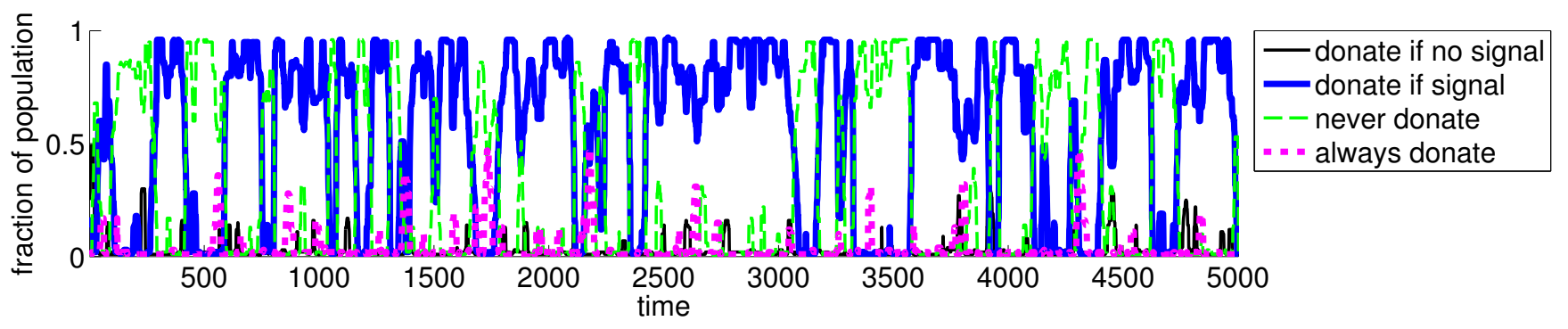

(b) Donor Population

Figure 3: Figure 3(a) and 3(b) show the distribution of strategies over time for populations initialized with uniform population distributions. $r$ is constant throughout the run.

over time initialized with uniform population distributions. The parameter set used to generate these plots is $a=0.8558$, $b=0.1287, c=0.2885, d=0.2637$ and $m=0.1609$ and $r$ $=0.5928$. Dominant signaler strategies oscillate between $S T$ and $S N$, and donor strategies oscillate between $D S$ and $D N$. Even though there are oscillations, $S T$ and $D S$ are dominant for a greater number of generations. The histograms in Figure 5(a) confirm this observation on a greater scale. For these plots, we kept a count of the dominant strategy for all repeats for every parameter set initialized with a uniform population distribution. The dominant strategies over time are $S T$ and $D S$ (Figure 5(a)).

\subsection{Evolution of Tags in SPS}

In this set of experiments, selection for strategies is muted and we only consider the evolution of tags with signaling, non-signaling and uniform population distributions. All tags are initialized with random numbers drawn from a uniform distribution. Since inclusive fitness is incorporated into the SPS game, the following equation of Hamilton's Rule applied to signaling equilibria holds

$$
r>\frac{d}{a-c}
$$

Figure 2 shows the p-value of expected relatedness against Hamilton's Rule applied to signaling equilibria for all 25 parameter sets. The p-values are calculated by stacking up all repeats for each parameter set, and determining the time each population spends with an expected relatedness that lies within the signaling equilibrium. Expected Relatedness is defined as

$$
E(r)=1-\frac{\sum_{t_{D}, t_{S}}\left|t_{D}-t_{S}\right|}{P^{2}},
$$

where $t_{D}$ is taken over the tag values in the donor population, $t_{S}$ is taken over the tag values in the signaler pop- ulation, and $\mathrm{P}$ is population size. It is used to describe the average relatedness between two populations. Figure 2 shows a decrease in p-value as the cost to benefit ratio of signaling increases. If the $r$ value isn't within the range of the signaling equilibria such that the aforementioned equations hold, the p-value decreases and the population has fewer individuals playing $S T$ and $D S$ strategies throughout the 5000 generations. This means that the difference in tag values is outside of the ideal range of $r$ values for signaling.

Apart from initializing tags with random numbers from a uniform distribution, we also tried different initialization techniques. Among these are tags with random numbers from a Gaussian distribution, tags with the same random number for the donors and another number for the signalers, tags with a value of 0.5 for both populations such that every member is related, and tags with a value of 1 for the signalers and 0 for the donors such that both populations are completely unrelated. Finally, for both populations, we also initialized tags with the ideal $r$ value for signaling to emerge. Similar results to Figure 2 were observed.

\subsection{Coevolution of Tags and Strategies}

In this experiment, both strategies and tags are allowed to coevolve, with mutation rates of 0.05 and 0.10 , respectively. Median filtering with a window size of 25 has been used to smooth Figures 4(a) and 4(b), which show the change in distribution of strategies over time for the signaler and donor populations. Figure $4(\mathrm{c})$ is a plot of expected relatedness over time, and the dotted lines denote the upper and lower bound of the acceptable range of $r$ values for signaling equilibria. Initial populations start from uniform origins, and the parameter set is $a=0.8558, b=0.1287, c=0.2885$, $d=0.2637$ and $m=0.1609$. Even with this example, we can observe that $S T$ and $D S$ strategies take over their own populations for brief periods of time, and the expected re- 


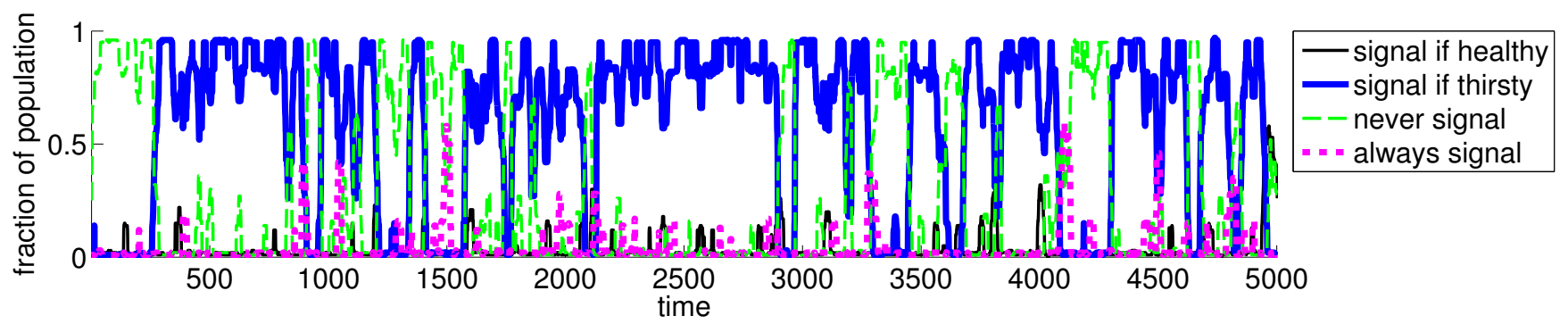

(a) Signaler Population

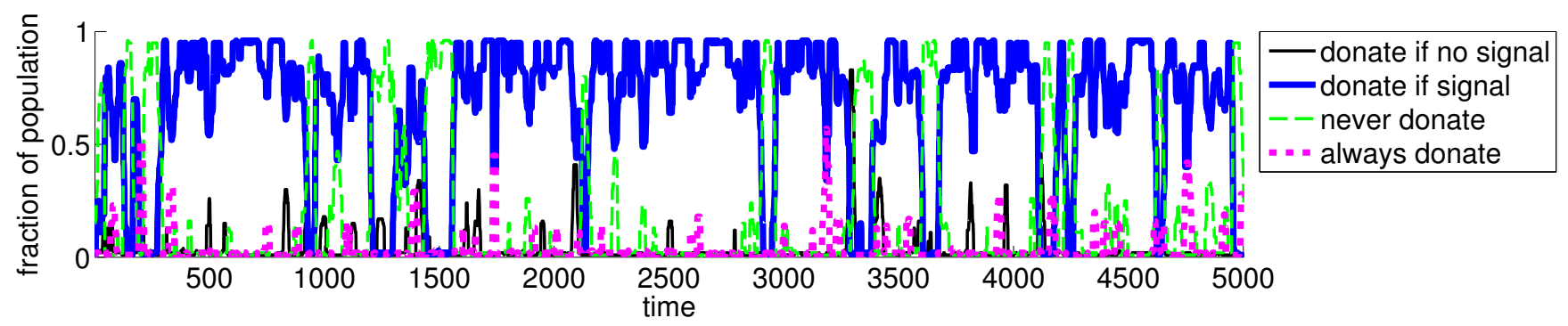

(b) Donor Population

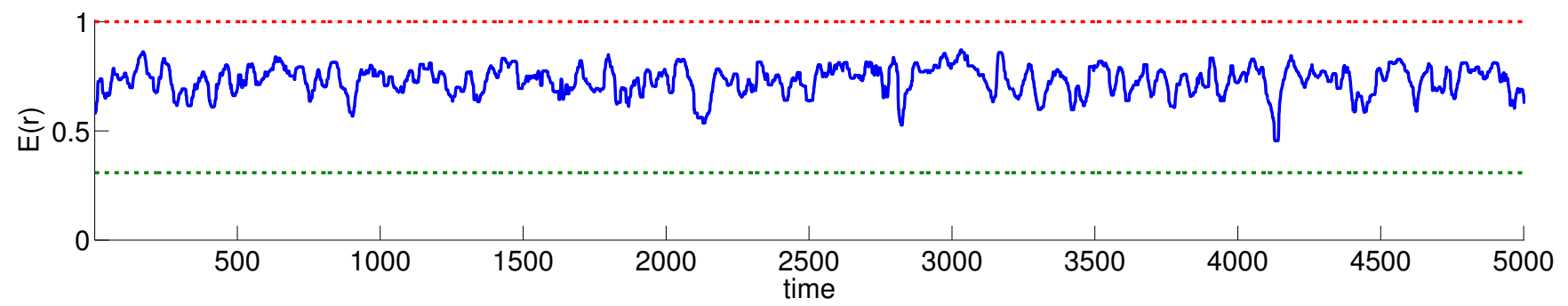

(c) Expected Relatedness of the Population

Figure 4: Figure 4(a) and 4(b) show the distribution of strategies over time for populations initialized with uniform population distributions and uniformly distributed tag values. Tags and strategies are coevolved. Horizontal lines on Figure 4(c) denote the lower and upper bound of $r$ for signalling equilibria. Note that $r$ is always within the range, but signaling is not constant.

latedness falls within the range of the signaling equilibria for the entire run. These plots resemble those in Figure 3, and we cannot see a pronounced effect of tags.

Figure 5(b) show the dominant strategies over all runs initialized with uniform population distributions. This was calculated by counting the number of generations in which a strategy was dominant. For the signalers, $S N$ and $S T$ are the two common strategies that take over the population. For the donors, $D N$ and $D S$ are the strategies that are dominant for the majority of time. However, both histograms suggest that $S N$ and $D N$ are the primary strategies that emerge. Especially when we compare the subplots in Figure 5 , we can see that tags, instead of prolonging the dominance of $S T$ and $D S$, shift the system towards uncommunicative populations. This suggests that tags might not be a sufficient mechanism to ensure the establishment of the honest signaling equilibrium under all parameter configurations.

In this experiment, we replaced tags with values randomly chosen from a uniform distribution for tag mutation. To confirm the validity of our results, we changed the implementation of tag mutation. Instead of replacing tag values, a value randomly chosen from a Gaussian distribution was added to the current tag value of the individual modulo 1.0. This implementation, based on the Gaussian mutation used in [4], yielded similar results to those in Figure 4 and 5(b).

\section{CONCLUSION}

In this study tags are implemented similarly to previous studies on the emergence of tag-based cooperation [17]; however, tags are used to directly encode the relatedness component of inclusive fitness in the SPS game. Inclusive fitness has been a long standing foundation of kin selection, and the corresponding explanations of evolved cooperative behaviors [7]. We find that, under parameter configurations which have been analytically and empirically determined to lead to honest signaling, populations with coevolving tagbased relatedness and player strategies only reach honest signaling equilibria for a subset of these verified parameter configurations. This supports recent arguments regarding the limitation of inclusive fitness theory [15] as a sufficient evolutionary mechanism to consistently explain the evolution of cooperation in the context of honest signaling. This suggests that the power of tag-based mechanisms may not simply be a result of their role in kin selection. 


\section{FUTURE WORK}

Future work for this research include analyzing other Nash equilibria in the game, particularly inverse honest signaling, defined as the majority of signalers playing $S H$ and donors playing $D Q$. Knowing the basins of attraction with respect to each parameter set, can help us choose parameters more carefully and favor runs where communication is likely to emerge. Additionally, studies should investigate the effect of tags on the dynamic stability and basins of attraction in the SPS game.

\section{Acknowledgements}

We thank Jordan Pollack and Lee Spector for commentary. We also acknowledge the Brandeis HPC for computing support.

\section{REFERENCES}

[1] C. Bergstrom and M. Lachmann. Signalling among relatives. I. Is costly signalling too costly? Philosophical Transactions of the Royal Society of London. Series B: Biological Sciences, 352(1353):609-617, 1997.

[2] B. Brilot and R. Johnstone. The limits to cost-free signalling of need between relatives. Proceedings of the Royal Society of London. Series B: Biological Sciences, 270(1519):1055-1060, 2003.

[3] B. Edmonds. The Emergence of Symbiotic Groups Resulting from Skill-Differentiation and Tags, Jan. 2006.

[4] G. Greenwood. Enhanced cooperation in the N-person iterated snowdrift game through tag mediation. In IEEE Conference on Computational Intelligence and Games (CIG), pages 1-8, 2011.

[5] D. Hales. Cooperation without memory or space: Tags, groups and the prisoner's dilemma. In Multi-agent-based simulation, volume 1979, pages 157-166. 2001.

[6] D. Hales. Altruism For Free using Tags. Paris ECCS, 2005.

[7] W. Hamilton. The genetical evolution of social behaviour. II. Journal of theoretical biology, 7(1):17-52, 1964.

[8] K. I. Harrington, A. P. Ozisik, and J. B. Pollack. The Effects of Finite Populations and Selection on the Emergence of Signaling. In Proceedings of Artificial Life XIII, page accepted, 2012.
[9] J. Holland. The effect of labels (tags) on social interactions. 1993.

[10] P. Hurd. Communication in discrete action-response games. Journal of Theoretical Biology, 174(2):217-222, 1995.

[11] S. Huttegger and K. Zollman. Dynamic stability and basins of attraction in the Sir Philip Sidney game. Proceedings of the Royal Society B: Biological Sciences, 277(1689):1915-1922, 2010.

[12] R. Johnstone and A. Grafen. The continuous Sir Philip Sidney game: a simple model of biological signalling. Journal of Theoretical Biology, 156(2):215-234, 1992.

[13] M. Lachmann and C. Bergstrom. Signalling among Relatives:: II. Beyond the Tower of Babel. Theoretical Population Biology, 54(2):146-160, 1998.

[14] M. Mitchell. An Introduction to Genetic Algorithms. MIT Press, 1996.

[15] M. Nowak. The evolution of eusociality. Nature, 466(7310):1057-1062, 2010.

[16] C. Rauter and A. Mooref. Do honest signalling models of offspring solicitation apply to insects? Proceedings of the Royal Society of London. Series B: Biological Sciences, 266(1429):1691-1696, 1999.

[17] R. L. Riolo, M. D. Cohen, and R. Axelrod. Evolution of cooperation without reciprocity. Nature, 414(6862):441-3, Nov. 2001.

[18] K. Sigmund and J. Hofbauer. Evolutionary games and population dynamics. Cambridge Univ. press. UK, 1998.

[19] J. Smith. Honest signalling: The Philip Sidney game. Animal Behaviour, 42:1034-1035, 1991.

[20] L. Spector and J. Klein. Genetic stability and territorial structure facilitate the evolution of tag-mediated altruism. Artificial Life, 12(4):553-560, 2006.

[21] L. Spector, J. Klein, C. Perry, and M. Feinstein. Emergence of Collective Behavior in Evolving Populations of Flying Agents. Genetic Programming and Evolvable Machines, 6(1):111-125, Mar. 2005.

[22] K. Wagner, J. Reggia, J. Uriagereka, and G. Wilkinson. Progress in the simulation of emergent communication and language. Adaptive Behavior, 11(1):37-69, 2003.

[23] E. Wilson. The insect societies. The insect societies., 1971.

[24] A. Zahavi. Mate selection-a selection for a handicap. Journal of theoretical Biology, 53:205-214, 1975. 

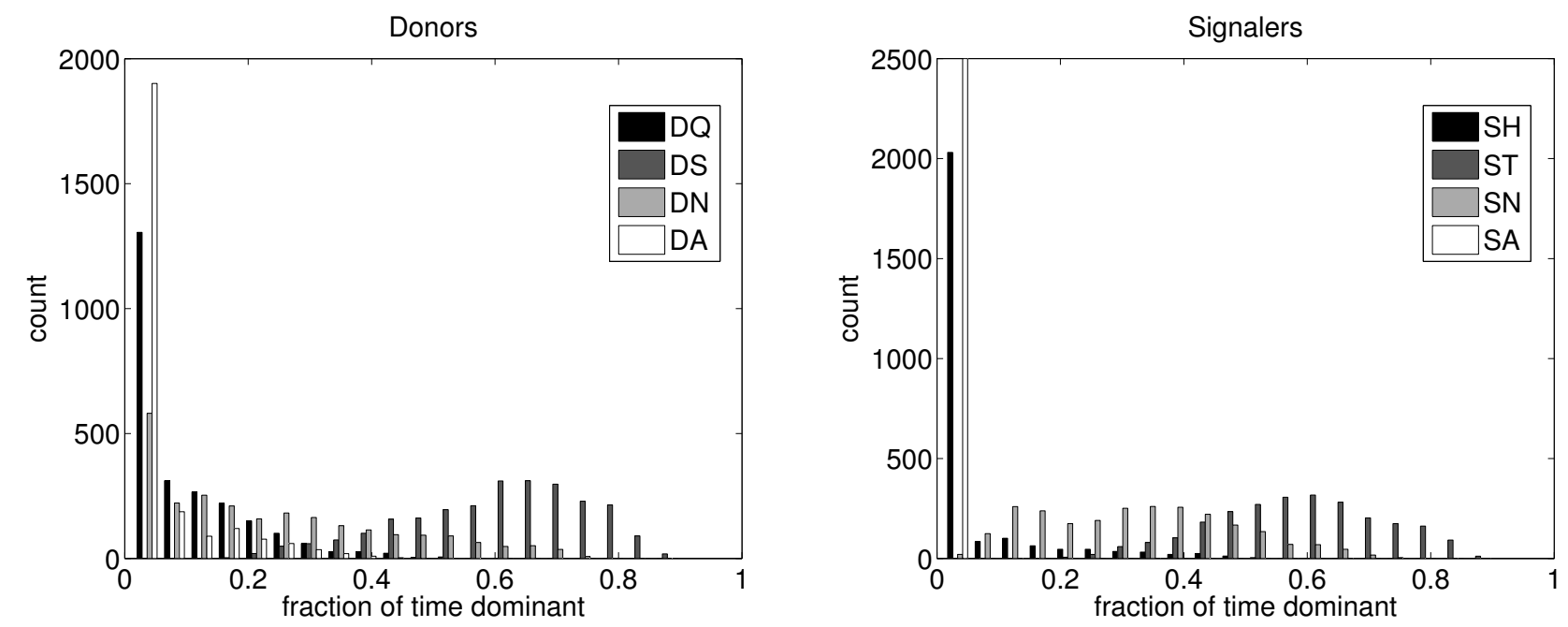

(a) Histograms with constant $r$ values. Note that $S T$ and $D S$ are the dominant strategies for signalers and donors, respectively.
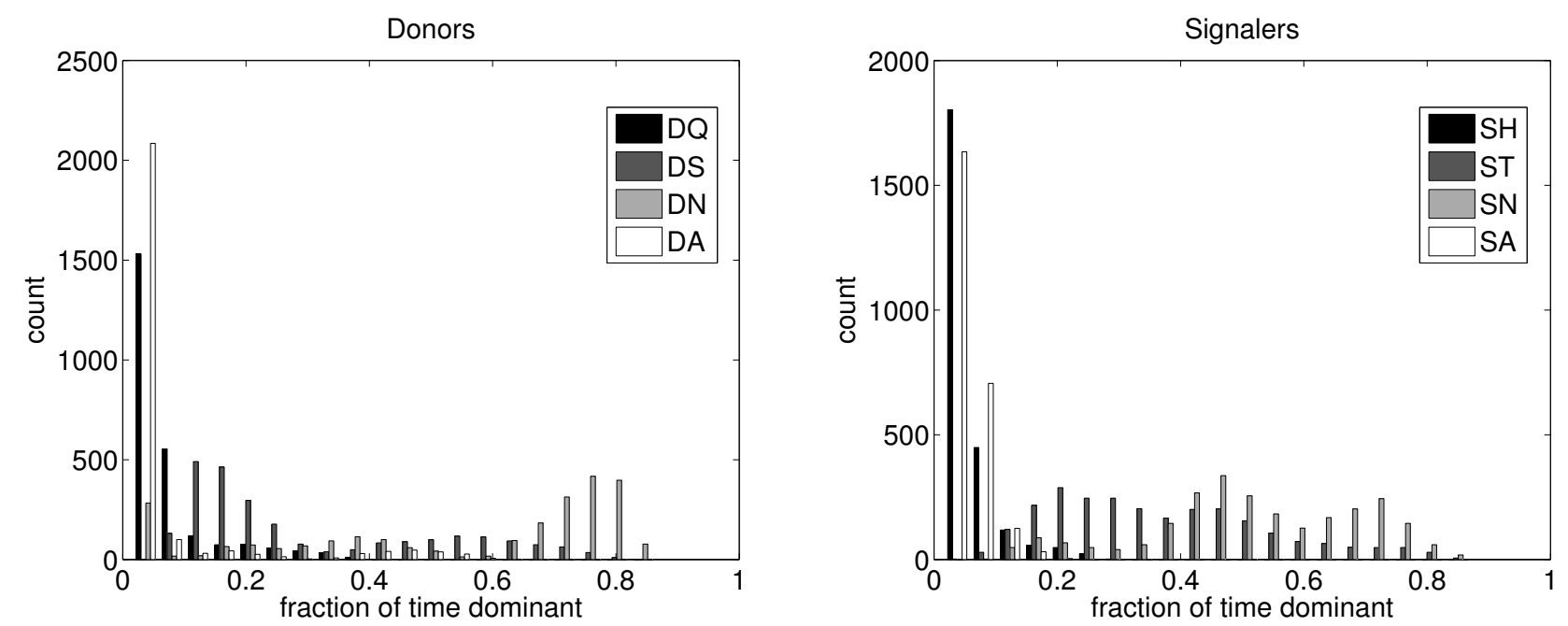

(b) Histograms with evolving strategies and tag values. Note that $S N$ and $D N$ are the dominant strategies for signalers and donors, respectively.

Figure 5: Histograms show the count of dominant strategies over generations for uniform population distributions. $S T$ and $D S$ dominate when strategies are evolved and tags are not. $S N$ and $D N$ are dominant when both tags and strategies are evolved. 